УДК $517.55+517.962 .26$

\title{
Multidimensional Analog of the Bernoulli Polynomials and its Properties
}

\author{
Olga A. Shishkina* \\ Institute of Mathematics and Computer Science \\ Siberian Federal University \\ Svobodny, 79, Krasnoyarsk, 660041
}

Russia

Received 07.04.2016, received in revised form 18.05.2016, accepted 20.06.2016

We consider a generalization of the Bernoulli numbers and polynomials to several variables, namely, we define the Bernoulli numbers associated with a rational cone and the corresponding Bernoulli polynomials. Also, we prove some properties of the Bernoulli polynomials.

Keywords: Bernoulli numbers and polynomials, generating functions, Todd operator, rational cone. DOI: 10.17516/1997-1397-2016-9-3-384-392.

\section{Introduction}

The Bernoulli numbers $b_{\mu}$ are the coefficients of the Taylor series expansion of the function $T(\xi)=\frac{\xi}{e^{\xi}-1}$ :

$$
T(\xi)=\sum_{\mu \geqslant 0} b_{\mu} \frac{\xi^{\mu}}{\mu !} .
$$

The Bernoulli numbers were introduced by J. Bernoulli in connection with the problem of summation of powers of consecutive integers: $1^{\mu}+2^{\mu}+\ldots+x^{\mu}$.

The Bernoulli polynomials

$$
B_{\mu}(x)=\sum_{k=0}^{\mu} C_{\mu}^{k} b_{\mu-k} x^{k}, \quad C_{\mu}^{k}=\frac{\mu !}{k !(\mu-k) !},
$$

where $b_{\mu}=B_{\mu}(0)$ are the Bernoulli numbers were considered by J. Bernoulli [1] for natural $x$, and for any $x$ these polynomials were first studied by Euler [2] who used the generating function in 1738:

$$
\frac{\xi}{e^{\xi}-1} e^{x \xi}=\sum_{\mu \geqslant 0} B_{\mu}(x) \frac{\xi^{\mu}}{\mu !} .
$$

Also J.L. Raabe (1801-1859) [3] studied the Bernoulli polynomials, he found two important formulas and introduced this term (J.L.Raabe, 1851). The Bernoulli and Euler polynomials were later systematically studied by N. Nörlund [4].

The Bernoulli numbers have wide applications in computer technology [5], combinatorial analysis [6,7] and in numerical analysis [8]. Gould [9] remarks that many sums involving binomial coefficients greatly benefit from the use of Bernoulli numbers. There is a number of papers about

*olga_a_sh@mail.ru

(C) Siberian Federal University. All rights reserved 
different generalizations of Bernoulli numbers and polynomials [10-15]. For example, Temme [16] uses generalized Bernoulli polynomials with complex powers.

In 1880 Appell (1855-1930) [17] characterized certain polynomial sequences $B_{\mu}(x)$ by the property $D B_{\mu}(x)=\mu B_{\mu-1}(x)$. Polynomials satisfying this condition are called Appell polynomials. For the Bernoulli polynomials this formula can be called the differentiation formula.

It was Euler who first defined a differential operator of infinite order $\frac{D}{e^{D}-1}=\sum_{\mu \geqslant 0} b_{\mu} \frac{D^{\mu}}{\mu !}$, where $D$ is a differential operator, now this operator is called the Todd operator and denoted $T d(D)$. It connects the solutions of the difference equation $f(x+1)-f(x)=\varphi(x)$ and the differential equation $D f(x)=T d(D) \varphi(x)$. The Bernoulli polynomials can be considered as a result of the Todd operator action on the monomial $x^{\mu}$ :

$$
B_{\mu}(x)=\frac{D}{e^{D}-1} x^{\mu} .
$$

The following identities hold for the Bernoulli polynomials: the argument addition formula

$$
B_{\mu}(x+y)=\sum_{k=0}^{\mu} C_{\mu}^{k} B_{k}(x) y^{\mu-k}
$$

the differentiation formula

$$
B_{\mu}^{\prime}(x)=\mu B_{\mu-1}(x)
$$

the complement formula

$$
B_{\mu}(x)=(-1)^{\mu} B_{\mu}(1-x)
$$

the multiplication formula

$$
B_{\mu}(m x)=m^{\mu-1} \sum_{k=0}^{\mu-1} B_{\mu}\left(x+\frac{k}{m}\right) .
$$

In this article we consider a generalization of the Bernoulli numbers and polynomials to the case of several variables, namely, we define the Bernoulli numbers associated with a rational cone, and the corresponding Bernoulli polynomials. For functions in several variables we construct a Todd operator associated with a rational cone $K$, and prove (Theorem 1) that the Bernoulli polynomials are the result of the Todd operator action on monomials. Further on, we formulate and prove multidimensional analogs of formulas (2)-(5) for the Bernoulli polynomials.

\section{A Todd operator and the Bernoulli polynomials in several variables}

A multidimensional analog of the Euler differential operator of infinite order $\frac{D}{e^{D}-1}$ is called the Todd operator (see, for example, [18]). The Todd operator [19] helps to connect volumes and a number of lattice points of convex polytopes. This formula is closely related to the HirzebruchRiemann-Roch theorem for smooth projective toric varieties. Note the articles [20-23] in which the operator $\frac{D_{1}}{e^{a_{1} D_{1}}-1} \ldots \frac{D_{n}}{e^{a_{n} D_{n}}-1}$, where $a_{j}$ are some constants, is considered.

Now we define the Bernoulli numbers, polynomials and a Todd operator associated with a rational cone $K$. Let $a^{1}, \ldots, a^{n}$ be linearly independent vectors with integer coordinates $a^{j}=$ 
$\left(a_{1}^{j}, \ldots, a_{n}^{j}\right), a_{i}^{j} \in \mathbb{Z}$. A rational cone generated by the vectors $a^{1}, \ldots, a^{n}$, is the set $K=\{y \in$ $\left.\mathbb{R}^{n}: y=\lambda_{1} a^{1}+\cdots+\lambda_{n} a^{n}, \lambda_{j} \in \mathbb{R}_{+}, j=1, \ldots, n\right\}$.

Note that such cone is simplicial, i.e. each element of the cone is represented by the cone generators in a unique way.

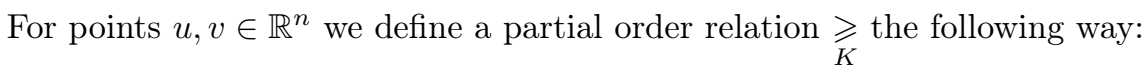

$$
u \underset{K}{\geqslant} v \Leftrightarrow u \in v+K,
$$

where $v+K$ is a shift of the cone $K$ by the vector $v$. Besides, let us write $u \ngtr v$, if $u \in K \backslash\{v+K\}$, i.e. if the condition $u \geqslant v$ is not fulfilled. Let $\mathbb{Z}^{n}=\mathbb{Z} \times \cdots \times \mathbb{Z}$, and note that each element $y \in K \cap \mathbb{Z}^{n}$ can be expressed as a linear combination of the basis vectors $y=\lambda_{1} a^{1}+\cdots+\lambda_{n} a^{n}$, $\lambda_{1} \geqslant 0, \ldots, \lambda_{n} \geqslant 0$. We can write it in a matrix form $y=A \lambda$, where $y$ and $\lambda$ are column vectors, $A$ is a matrix with the determinant $\Delta \neq 0$, and the columns are the coordinates of vectors $a^{j}$

$$
A=\left(\begin{array}{ccc}
a_{1}^{1} & \ldots & a_{1}^{n} \\
. . & . . & . . \\
a_{n}^{1} & \ldots & a_{n}^{n}
\end{array}\right) .
$$

For $j=1, \ldots, n$ consider the hyperplanes

$$
L_{j, k}=\left\{\xi:\left\langle a^{j}, \xi\right\rangle=2 k \pi i\right\},
$$

where $i=\sqrt{-1}, j=1, \ldots, n, k=0, \pm 1, \pm 2 \ldots$, and for a meromorphic function

$$
T(\xi)=\prod_{j=1}^{n} \frac{\left\langle a^{j}, \xi\right\rangle}{e^{\left\langle a^{j}, \xi\right\rangle}-1},
$$

where $\left\langle a^{j}, \xi\right\rangle=\sum_{k=1}^{n} a_{k}^{j} \xi_{k}, \xi=\left(\xi_{1}, \ldots, \xi_{n}\right)$, the hyperplanes $L_{j, 0}, j=1, \ldots, n$ are 'removable' singular set, so the function $T(\xi)$ is holomorphic in a neighborhood of the origin, more exactly for $R=2 \pi / \max _{j}\left\|a^{j}\right\|$ it is holomorphic in a polycylinder $U_{R}=\left\{\xi:\left|\xi_{j}\right|<R, j=1, \ldots, n\right\}$, and for $k=0, \pm 1, \pm 2 \ldots$ the hyperplanes $L_{j, k}$ do not cross with the polycylinder $U_{R}$. Therefore, in this polycylinder the function $T(\xi)$ expands into a series

$$
T(\xi)=\sum_{\mu \geqslant 0} \frac{b_{\mu}^{A}}{\mu !} \xi^{\mu}
$$

where $\mu=\left(\mu_{1}, \ldots, \mu_{n}\right), \mu !=\mu_{1} ! \ldots \mu_{n} !, \xi^{\mu}=\xi_{1}{ }^{\mu_{1}} \ldots \xi_{n}{ }^{\mu_{n}}$, and $\mu \geqslant 0$ means $\mu_{j} \geqslant 0, j=$ $1, \ldots, n$

The cofficients $b_{\mu}^{A}$ of the series (6) we call the Bernoulli number associated with the rational cone .

For $\mu=\left(\mu_{1}, \ldots, \mu_{n}\right)$ the Bernoulli polynomials in several variables are the polynomials

$$
B_{\mu}^{A}(x)=\sum_{0 \leqslant k \leqslant \mu} \frac{\mu !}{(\mu-k) ! k !} b_{\mu-k}^{A} x^{k},
$$

where $b_{k}^{A}$ are the Bernoulli numbers, $k=\left(k_{1}, \ldots, k_{n}\right), x=\left(x_{1}, \ldots, x_{n}\right)$, $\mu-k=\left(\mu_{1}-k_{1}, \ldots, \mu_{n}-k_{n}\right)$. 
For $n=1$ and $a=1$ thus defined Bernoulli numbers and polynomials coincide with the classical ones.

Example 1. For the cone $K$ generated by vectors $a^{1}=(1,0)$ and $a^{2}=(1,1)$ the first Bernoulli numbers are $b_{00}=1, b_{10}=-1, b_{01}=-\frac{1}{2}, b_{11}=\frac{5}{12}$, and for $\mu=(1,1)$ the Bernoulli polynomials are $B_{11}^{A}\left(x_{1}, x_{2}\right)=x_{1} x_{2}-\frac{1}{2} x_{1}-x_{2}+\frac{5}{12}$.

A Todd mapping is the meromorphic function $T d^{A}: \mathbb{C}^{n} \rightarrow \mathbb{C}$ defined by $T d^{A}(\xi)=$ $\prod_{j=1}^{n} \frac{\left\langle a^{j}, \xi\right\rangle}{e^{\left\langle a^{j}, \xi\right\rangle}-1}$. It expands into a series $T d^{A}(\xi)=\sum_{\mu \geqslant 0} \frac{b_{\mu}^{A}}{\mu !} \xi^{\mu}$, and the Todd operator is the result of substitution of the differential operator $\partial=\left(\partial_{1}, \ldots, \partial_{n}\right)$ in place of $\xi$ :

$$
T d^{A}(\partial)=\sum_{\mu \geqslant 0} \frac{b_{\mu}^{A}}{\mu !} \partial^{\mu}
$$

where $\mu \geqslant 0$ means $\mu_{j} \geqslant 0, j=1, \ldots, n$.

Remark 1. In general, to define the Bernoulli numbers and polynomials and the Todd operator we do not need the condition of rationality of the cone, i.e., any real numbers can be the coordinates of the vectors $a^{j}$. However, in the problem of summation of functions the rationality of the cone is a natural condition.

The Todd operator can act on a function $h$ if the series $T d(\partial) h$ converges absolutely in the domain of the function $h$ uniformly on compact subsets. It is obvious that polynomials can be acted on by the Todd operator and for the monomial $x^{\mu}=x_{1}^{\mu_{1}} \ldots x_{n}^{\mu_{n}}$ we have the following analog of formula (1).

Theorem 1.1. If $T d^{A}(\partial)$ is the Todd operator and $B_{\mu}^{A}(x)$ are Bernoulli polynomials associated with a rational cone then we have the equality

$$
\operatorname{Td}^{A}(\partial) x^{\mu}=B_{\mu}^{A}(x)
$$

Proof. First note that the equality $\frac{\partial^{\alpha} x^{\mu}}{\alpha !}=\frac{\mu !}{\alpha !(\mu-\alpha) !} x^{\mu-\alpha}$ holds true. Indeed,

$$
\begin{array}{r}
\frac{\partial^{\alpha} x^{\mu}}{\alpha !}=\frac{\mu_{1}\left(\mu_{1}-1\right) \ldots\left(\mu_{1}-\alpha_{1}+1\right)}{\alpha_{1} !} \ldots \frac{\mu_{n}\left(\mu_{n}-1\right) \ldots\left(\mu_{n}-\alpha_{n}+1\right)}{\alpha_{n} !} x^{\mu_{1}-\alpha_{1}} \ldots x^{\mu_{n}-\alpha_{n}}= \\
=\frac{\mu !}{\alpha !(\mu-\alpha) !} x^{\mu-\alpha} .
\end{array}
$$

Next, using the definition of the Todd operator and the previous equality we obtain

$$
\begin{gathered}
\operatorname{Td}(\partial) x^{\mu}=\prod_{j=1}^{n} \frac{\left\langle a^{j}, \partial\right\rangle}{e^{\left\langle a^{j}, \partial\right\rangle}-1} x^{\mu}=\sum_{\alpha \geqslant 0} \frac{b_{\alpha}^{A}}{\alpha !} \partial^{\alpha} x^{\mu}= \\
=\sum_{0 \leqslant \alpha \leqslant \mu} b_{\alpha}^{A} \frac{\mu !}{\alpha !(\mu-\alpha) !} x^{\mu-\alpha}=\sum_{0 \leqslant k \leqslant \mu} b_{\mu-k}^{A} \frac{\mu !}{k !(\mu-k) !} x^{k}=B_{\mu}^{A}(x) .
\end{gathered}
$$




\section{Complement, differentiation, addition, and multiplication formulas}

To formulate an analog of the property of differentiation of Bernoulli polynomials $B_{\mu}^{A}(x)$ we need a differential operator in the direction of the vectors $a^{j}$ generating the cone $K$ :

$$
D_{j}=\left\langle a^{j}, \partial\right\rangle=\sum_{k=1}^{n} a_{k}^{j} \partial_{k}
$$

where $\partial_{j}$ are operators of differentiating with respect to variables, $\partial=\left(\partial_{1}, \ldots, \partial_{n}\right), \partial^{\mu}=$ $\partial_{1}^{\mu_{1}} \ldots \partial_{n}^{\mu_{n}}, j=1, \ldots, n$. Denote $D=D_{1} \ldots D_{n}$.

Theorem 2.1. The following multidimensional analogs of the properties (2)-(5) of Bernoulli polynomials hold true:

1) the argument addition formula

$$
B_{\mu}^{A}(x+y)=\sum_{0 \leqslant k \leqslant \mu} \frac{\mu !}{k !(\mu-k) !} B_{k}^{A}(x) y^{\mu-k} ;
$$

2) the differentiation formula

$$
D B_{\mu}^{A}(x)=\sum_{0 \leqslant k \leqslant \mu,\|k\|=n} \frac{\mu !}{k !(\mu-k) !} B_{\mu-k}^{A}(x) \frac{M_{k}}{k !},
$$

where $M_{k}$ are the coefficients of the polynomial

$$
\prod_{j=1}^{n}\left\langle a^{j}, \xi\right\rangle=\sum_{\|k\|=n} M_{k} \frac{\xi^{k}}{k !}
$$

3) the complement formula

$$
B_{\mu}^{A}(x)=(-1)^{\|\mu\|} B_{\mu}^{A}(a-x),
$$

where $a=a^{1}+\cdots+a^{n},\|\mu\|=\mu_{1}+\cdots+\mu_{n}$;

4) the multiplication formula

$$
B_{\mu}^{A}(m x)=m^{\mu-I} \sum_{0 \leqslant k \leqslant \mu-I} B_{\mu}^{A}\left(x+\frac{1}{m} A k\right),
$$

where $I=(1, \ldots, 1), \frac{1}{m}=\left(\frac{1}{m_{1}}, \ldots, \frac{1}{m_{n}}\right)$ is a row vector, $k=\left(\begin{array}{c}k_{1} \\ . . \\ k_{n}\end{array}\right)$ is a column vector.

Proof. To prove this theorem it is convenient to use the definition of Bernoulli polynomials via generating function

$$
\prod_{j=1}^{n} \frac{\left\langle a^{j}, \xi\right\rangle}{e^{\left\langle a^{j}, \xi\right\rangle}-1} e^{\langle x, \xi\rangle}=\sum_{\mu \geqslant 0} B_{\mu}^{A}(x) \frac{\xi^{\mu}}{\mu !}
$$

where $\langle x, \xi\rangle=x_{1} \xi_{1}+\cdots+x_{n} \xi_{n}$. 
1. The proof of the argument addition formula. Using the definition of Bernoulli polynomials via generating function we obtain

$$
\sum_{\mu \geqslant 0} B_{\mu}^{A}(x+y) \frac{\xi^{\mu}}{\mu !}=\prod_{j=1}^{n} \frac{\left\langle a^{j}, \xi\right\rangle}{e^{\left\langle a^{j}, \xi\right\rangle}-1} e^{\langle x+y, \xi\rangle}=\prod_{j=1}^{n} \frac{\left\langle a^{j}, \xi\right\rangle}{e^{\left\langle a^{j}, \xi\right\rangle}-1} e^{\langle x, \xi\rangle} e^{\langle y, \xi\rangle}=\sum_{k \geqslant 0} B_{k}^{A}(x) \frac{\xi^{k}}{k !} \sum_{\alpha \geqslant 0} y^{\alpha} \frac{\xi^{\alpha}}{\alpha !} .
$$

Multiplying power series and collecting similar terms, we get

$$
\sum_{\mu \geqslant 0} B_{\mu}^{A}(x+y) \frac{\xi^{\mu}}{\mu !}=\sum_{\mu \geqslant 0}\left(\sum_{k+\alpha=\mu} \frac{\mu !}{k ! \alpha !} B_{k}^{A}(x) y^{\alpha}\right) \frac{\xi^{\mu}}{\mu !}=\sum_{\mu \geqslant 0}\left(\sum_{0 \leqslant k \leqslant \mu} \frac{\mu !}{k !(\mu-k) !} B_{k}^{A}(x) y^{\mu-k}\right) \frac{\xi^{\mu}}{\mu !} .
$$

The formula follows from equality of coefficients at identical powers of $\xi$.

2. The proof of the differentiation formula. Act by the operator $D$ on both parts of the equality

$$
\sum_{\mu \geqslant 0} B_{\mu}^{A}(x) \frac{\xi^{\mu}}{\mu !}=\prod_{j=1}^{n} \frac{\left\langle a^{j}, \xi\right\rangle}{e^{\left\langle a^{j}, \xi\right\rangle}-1} e^{\langle x, \xi\rangle}
$$

to obtain, keeping in mind that $D e^{\langle x, \xi\rangle}=e^{\langle x, \xi\rangle} \prod_{j=1}^{n}\left\langle a^{j}, \xi\right\rangle$,

$$
\sum_{\mu \geqslant 0} D B_{\mu}^{A}(x) \frac{\xi^{\mu}}{\mu !}=\prod_{j=1}^{n} \frac{\left\langle a^{j}, \xi\right\rangle}{e^{\left\langle a^{j}, \xi\right\rangle}-1} e^{\langle x, \xi\rangle} \prod_{j=1}^{n}\left\langle a^{j}, \xi\right\rangle .
$$

Let $M_{k}$ be the coefficients of the polynomial $\prod_{j=1}^{n}\left\langle a^{j}, \xi\right\rangle$ at $\frac{\xi^{k}}{k !}$ :

$$
\prod_{j=1}^{n}\left\langle a^{j}, \xi\right\rangle=\sum_{\|k\|=n} M_{k} \frac{\xi^{k}}{k !}
$$

then

$$
\sum_{\mu \geqslant 0} D B_{\mu}^{A}(x) \frac{\xi^{\mu}}{\mu !}=\sum_{\alpha \geqslant 0} B_{\alpha}^{A}(x) \frac{\xi^{\alpha}}{\alpha !} \sum_{\|k\|=n} M_{k} \frac{\xi^{k}}{k !} .
$$

Multiplying power series, we have

$$
\sum_{\mu \geqslant 0} D B_{\mu}^{A}(x) \frac{\xi^{\mu}}{\mu !}=\sum_{\mu \geqslant 0}\left(\sum_{\alpha+k=\mu,\|k\|=n} \frac{\mu !}{k ! \alpha !} B_{\alpha}^{A}(x) M_{k}\right) \frac{\xi^{\mu}}{\mu !} .
$$

After changing indices we equal the coefficients of powers of $\xi$ and obtain the necessary property.

3. The proof of the complement formula.

$$
\begin{gathered}
\sum_{\mu \geqslant 0}(-1)^{\|\mu\|} B_{\mu}^{A}(a-x) \frac{\xi^{\mu}}{\mu !}=\sum_{\mu \geqslant 0} B_{\mu}^{A}(a-x) \frac{(-\xi)^{\mu}}{\mu !}=\prod_{j=1}^{n} \frac{\left\langle a^{j},-\xi\right\rangle}{e^{\left\langle a^{j},-\xi\right\rangle}-1} e^{\langle a-x,-\xi\rangle}= \\
=\prod_{j=1}^{n} \frac{-\left\langle a^{j}, \xi\right\rangle}{\frac{1}{e^{\left\langle a^{j}, \xi\right\rangle}}-1} e^{\langle a-x,-\xi\rangle}=\prod_{j=1}^{n} \frac{\left\langle a^{j}, \xi\right\rangle}{1-\frac{1}{e^{\left\langle a^{j}, \xi\right\rangle}}} e^{\langle a-x,-\xi\rangle}= \\
=\prod_{j=1}^{n} \frac{\left\langle a^{j}, \xi\right\rangle}{\frac{e^{\left\langle a^{j}, \xi\right\rangle}}{e^{\left\langle a^{j}, \xi\right\rangle}}-\frac{1}{e^{\left\langle a^{j}, \xi\right\rangle}}} e^{\langle a-x,-\xi\rangle}=\prod_{j=1}^{n} \frac{\left\langle a^{j}, \xi\right\rangle}{e^{\left\langle a^{j}, \xi\right\rangle}-1} e^{\left\langle a^{j}, \xi\right\rangle} e^{-\langle a, \xi\rangle} e^{\langle x, \xi\rangle}
\end{gathered}
$$


Since $e^{-\langle a, \xi\rangle}=\prod_{j=1}^{n} e^{\left\langle a^{j}, \xi\right\rangle}$,

$$
\sum_{\mu \geqslant 0}(-1)^{\|\mu\|} B_{\mu}^{A}(a-x) \frac{\xi^{\mu}}{\mu !}=\prod_{j=1}^{n} \frac{\left\langle a^{j}, \xi\right\rangle}{e^{\left\langle a^{j}, \xi\right\rangle}-1} e^{\left\langle a^{j}, \xi\right\rangle} e^{-\left\langle a^{j}, \xi\right\rangle} e^{\langle x, \xi\rangle}=\sum_{\mu \geqslant 0} B_{\mu}^{A}(x) \frac{\xi^{\mu}}{\mu !} .
$$

Equal the coefficients at powers of $\xi$ to obtain the complement formula.

4. The proof of the multiplication formula. From the definition of Bernoulli polynomials we have

$$
\sum_{\mu \geqslant 0} B_{\mu}^{A}(m x) \frac{\xi^{\mu}}{\mu !}=\prod_{j=1}^{n} \frac{\left\langle a^{j}, \xi\right\rangle}{e^{\left\langle a^{j}, \xi\right\rangle}-1} e^{\langle m x, \xi\rangle} .
$$

Using the formula of geometric progression with denominator $e^{\left\langle a^{j}, \xi\right\rangle}$, we obtain

$$
\frac{1}{e^{\left\langle a^{j}, \xi\right\rangle}-1}=\frac{1+e^{\left\langle a^{j}, \xi\right\rangle}+\ldots+e^{\left\langle\left(m_{j}-1\right) a^{j}, \xi\right\rangle}}{e^{\left\langle m_{j} a^{j}, \xi\right\rangle}-1},
$$

After substituting this equality into the right hand side of (8) and after some transformations we obtain

$$
\begin{array}{r}
\sum_{\mu \geqslant 0} B_{\mu}^{A}(m x) \frac{\xi^{\mu}}{\mu !}=\prod_{j=1}^{n} \frac{1+e^{\left\langle a^{j}, \xi\right\rangle}+\ldots+e^{\left\langle\left(m_{j}-1\right) a^{j}, \xi\right\rangle}}{e^{\left\langle m_{j} a^{j}, \xi\right\rangle}-1}\left\langle m_{j} a^{j}, \xi\right\rangle m_{j}{ }^{-1} e^{\langle m x, \xi\rangle}= \\
=m^{-I} \prod_{j=1}^{n} \frac{\left\langle a^{j}, m_{j} \xi\right\rangle}{e^{\left\langle a^{j}, m_{j} \xi\right\rangle}-1} e^{\langle x, m \xi\rangle} \prod_{j=1}^{n}\left(1+e^{\left\langle a^{j}, \xi\right\rangle}+\ldots+e^{\left\langle\left(m_{j}-1\right) a^{j}, \xi\right\rangle}\right) .
\end{array}
$$

Since

$$
\prod_{j=1}^{n}\left(1+e^{\left\langle a^{j}, \xi\right\rangle}+\ldots+e^{\left\langle\left(m_{j}-1\right) a^{j}, \xi\right\rangle}\right)=\sum_{0 \leqslant k \leqslant m-I} e^{\left\langle k_{1} a^{1}+\ldots+k_{n} a^{n}, \xi\right\rangle}
$$

we get

$$
\begin{aligned}
& \sum_{\mu \geqslant 0} B_{\mu}^{A}(m x) \frac{\xi^{\mu}}{\mu !}=m^{-I} \prod_{j=1}^{n} \frac{\left\langle a^{j}, m_{j} \xi\right\rangle}{e^{\left\langle a^{j}, m_{j} \xi\right\rangle}-1} e^{\langle x, m \xi\rangle} \sum_{0 \leqslant k \leqslant m-I} e^{\left\langle k_{1} a^{1}+\ldots+k_{n} a^{n}, \xi\right\rangle}= \\
&=\sum_{0 \leqslant k \leqslant m-I} m^{-I} \prod_{j=1}^{n} \frac{\left\langle a^{j}, m_{j} \xi\right\rangle}{e^{\left\langle a^{j}, m_{j} \xi\right\rangle}-1} e^{\left\langle m x+k_{1} a^{1}+\ldots+k_{n} a^{n}, \xi\right\rangle} .
\end{aligned}
$$

Since $k_{1} a^{1}+\cdots+k_{n} a^{n}=A k$, where $A=\left(\begin{array}{ccc}a_{1}^{1} & \ldots & a_{1}^{n} \\ . . & . . & . . \\ a_{n}^{1} & \ldots & a_{n}^{n}\end{array}\right)$ and $k=\left(\begin{array}{c}k_{1} \\ . . \\ k_{n}\end{array}\right), \frac{1}{m}=$ $\left(\frac{1}{m_{1}}, \ldots, \frac{1}{m_{n}}\right)$, we obtain

$$
\begin{aligned}
& \sum_{\mu \geqslant 0} B_{\mu}^{A}(m x) \frac{\xi^{\mu}}{\mu !}=\sum_{0 \leqslant k \leqslant m-I} m^{-I} \prod_{j=1}^{n} \frac{\left\langle a^{j}, m_{j} \xi\right\rangle}{e^{\left\langle a^{j}, m_{j} \xi\right\rangle}-1} e^{\left\langle x+\frac{1}{m} A k, m \xi\right\rangle}= \\
& =m^{-I} \sum_{0 \leqslant k \leqslant m-I} \sum_{\mu \geqslant 0} B_{\mu}^{A}\left(x+\frac{1}{m} A k\right) m^{\mu} \frac{\xi^{\mu}}{\mu !}=\sum_{\mu \geqslant 0}\left(m^{\mu-I} \sum_{0 \leqslant k \leqslant m-I} B_{\mu}^{A}\left(x+\frac{1}{m} A k\right)\right) \frac{\xi^{\mu}}{\mu !} .
\end{aligned}
$$

Equal the coefficients at powers of $\xi$ to obtain the formula.

This work is supported by the Russian Federation Government grant to conduct research under the guidance of leading scientists at Siberian Federal University (contract 14. Y26.31.0006). 


\section{References}

[1] Jacob Bernoulli, Ars Conjectandi, Basel 1713.

[2] L.Euler, Institutiones calculi differentialis 1755, new printing Birkhäuser 1913.

[3] J.-L.Raabe, Die Jacob Bernoullische Funktion, Zürich, 1848.

[4] N.E.Nörlund, Differenzenrechnung, Berlin, 1924.

[5] R.L.Graham, D.E.Knuth, O.Patashnik, Concrete mathematics, A foundation for computer science, Second edition. Addison-Wesley Publishing Company, Reading, MA, 1994.

[6] J.Riordan, Combinatorial identities, Reprint of the 1968 original. Robert E. Krieger Publishing Co., Huntington, N.Y., 1979.

[7] G.-C.Rota, B.D.Taylor, The classical umbral calculus, SIAM J. Math. Anal., 25(1994), no. $2,694-711$.

[8] C.-E.Fröberg, Lärobok i numerisk analys, Stockholm, 1962.

[9] H.W.Gould, Combinatorial identities, Morgantown printing and binding co., Morgantown WV-USA, 1972.

[10] T.Ernst, $q$-Bernoulli and $q$-Euler polynomials, an umbral approach, International Journal of Difference Equations, 1(2006), no. 1, 31-80.

[11] L. Carlitz, Bernoulli and Euler numbers and orthogonal polynomials, Duke Math. J., 26(1959), 694-711.

[12] T. Ernst, The history of $q$-calculus and a new method, Uppsala, 2000.

[13] D.H.Lehmer, Lacunary recurrence formulas for the numbers of Bernoulli and Euler, Ann. of Math., 36(1935), no. 3, 637-649.

[14] H.M.Srivastava, A.Pintér, Remarks on some relationships between the Bernoulli and Euler polynomials, Appl. Math. Lett., 17(2004), no. 4, 375-380.

[15] H.S.Vandiver, Simple explicit expressions for generalized Bernoulli numbers of the first order, Duke Math. J., 8(1941), 575-584.

[16] N.M.Temme, Bernoulli polynomials old and new: Generalization and asymptotics, $C W I$ Quarterly, (1995), no. 1, 47-66.

[17] P.Appell, Sur une classe de polynômes, Annales Scientifiques de l'École Normale Supérieure Sér, 2(1880), no. 9, 119-144.

[18] M.Lenz, Lattice points in polytopes, box splines, and Todd operators, International Mathematics Research Notices, (2015), no. 14, 5289-5310.

[19] A.V.Pukhlikov, A.G.Khovanskii, The Riemann-Roch theorem for integrals and sums of quasipolynomials on virtual polytopes, St. Petersburg Mathematical Journal, 4 (1993), no. $4,789-812$. 
[20] M.Brion, M.Vergne, Lattice points in simple polytopes, Journal of the American Mathematical Society, 10(1997), no. 2, 371-392.

[21] M.Brion, M.Vergne, Residue formulae, vector partition functions and lattice points in rational polytopes, Journal of the American Mathematical Society, 10(1997), no. 4, 797-833.

[22] M.Vergne, Residue formulae for Verlinde sums, and for number of integral points in convex rational polytopes, European women in mathematics (Malta, 2001), World Sci. Publ., River Edge, NJ, 2003, 225-285.

[23] M.Brion, N.Berline, Local Euler-Maclaurin formula for polytopes, Moscow Mathematical Society Journal, 7(2007), 355-383.

\section{Многомерный аналог многочленов Бернулли и его свойства}

\section{Ольга А. Шишкина}

Институт математики и фундаментальной информатики Сибирский федеральный университет Свободный, 79, Красноярск, 660041

Россия

В работе рассматривается некоторое обобщение чисел и многочленов Бернулли на случай нескольких переменных, а именно определяются числа Бернулли, ассоциированные с рачиональным конусом, и соответствующие им многочлены Бернулли. Доказаны некоторые свойства многочленов Бернулли.

Ключевые слова: числа и многочлены Бернулли, производящие функиии, оператор Тодда, рачиональныц конус. 